\title{
NÍVEL DE CONFORTO NA DIMENSÃO SEGURANÇA DE FAMILIARES DE PACIENTES INTERNADOS EM UNIDADE DE TERAPIA INTENSIVA
}

\author{
Edineia Miranda Machado ${ }^{1}$ \\ Tatiana Brusamarello ${ }^{1}$
}

\author{
https://orcid.org/0000-0002-5767-196X \\ https://orcid.org/0000-0002-8060-8723
}

Objetivo: Verificar o nível de conforto na dimensão segurança de familiares de pacientes internados em leitos de Unidade de Terapia Intensiva. Método: Estudo quantitativo, descritivo, transversal, realizado no período de junho a setembro de 2019 , com 40 familiares de pacientes internados em leitos de Unidade de Terapia Intensiva de um hospital universitário do sul do Brasil. Os dados foram coletados por meio da Escala de Conforto para Familiares de Pessoas em Estado Crítico de Saúde e analisados por estatística descritiva simples. Resultados: Dos familiares entrevistados a maioria era do sexo feminino, com faixa etária de 40 a 59 anos, parentes de primeiro grau, com ensino médio completo. A média geral do nível de conforto em relação a dimensão segurança foi de 4,36 para Unidade de Terapia Intensiva I e 4,31 para a Unidade de Terapia Intensiva II, resultando em médio conforto. Conclusão: O nível de conforto na dimensão segurança mostrou-se médio, evidenciando reflexos positivos da inclusão familiar no contexto hospitalar relacionado ao acolhimento, interação com a equipe de saúde e confiabilidade no cuidado prestado.

Descritores: Cuidados de Enfermagem; Família; Cuidados Críticos.

\section{COMFORT LEVEL IN THE SAFETY DIMENSION OF FAMILY MEMBERS OF PATIENTS HOSPITALIZED IN INTENSIVE CARE UNIT}

Objective: To verify the level of comfort in the safety dimension of family members of patients admitted to beds in the Intensive Care Unit. Method: Quantitative, descriptive, cross-sectional study, carried out from June to September 2019 , with 40 family members of patients admitted to beds in the Intensive Care Unit of a university hospital in southern Brazil. The data were collected using the Comfort Scale for Family Members of People in Critical Health and analyzed using simple descriptive statistics. Results: Of the interviewed family members, most were female, aged 40 to 59 years old, first-degree relatives, with high school education. The general average of the comfort level in relation to the safety dimension was 4.36 for the Intensive Care Unit I and 4.31 for the Intensive Care Unit II, resulting in medium comfort. Conclusion: The level of comfort in the safety dimension was shown to be medium, showing positive reflexes of family inclusion in the hospital context related to welcoming, interaction with the health team and reliability in the care provided.

Descriptors: Nursing Care; Family; Critical Care.

\section{NIVEL DE CONFORT EN LA DIMENSIÓN DE SEGURIDAD DE LOS FAMILIARES MIEMBROS DE PACIENTES HOSPITALIZADOS EN LA UNIDAD DE ATENCIÓN INTENSIVA}

Objetivo: Verificar el nivel de confort en la dimensión de seguridad de los familiares de pacientes hospitalizados en camas de la Unidad de Cuidados Intensivos. Método: Estudio cuantitativo, descriptivo, transversal, realizado de junio a septiembre de 2019 , con 40 familiares de pacientes ingresados en camas en la Unidad de Cuidados Intensivos de un hospital universitario en el sur de Brasil. Los datos fueron recolectados usando la Escala de Confort para los miembros de la familia de personas en estado crítico de salud y analizados usando estadísticas descriptivas simples. Resultados: De los familiares entrevistados, la mayoría eran mujeres, de 40 a 59 años, familiares de primer grado, con educación secundaria. El promedio general del nivel de comodidad en relación con la dimensión de seguridad fue 4.36 para la Unidad de Cuidados Intensivos I y 4.31 para la Unidad de Cuidados Intensivos II, lo que resultó en un confort medio. Conclusión: Se demostró que el nivel de comodidad en la dimensión de seguridad era medio, mostrando reflejos positivos de inclusión familiar en el contexto hospitalario relacionados con la acogida, la interacción con el equipo de salud y la confiabilidad en la atención brindada.

Descriptores: Atención de Enfermería; Familia; Cuidados Críticos

${ }^{1}$ Complexo Hospital de Clínicas da Universidade Federal do Paraná, PR, Brasil.

Autor correspondente: Tatiana Brusamarello Email: brusamarello.tatiana@gmail.com Recebido: 19/2/20 Aceito: 15/5/20 


\section{INTRODUÇÃO}

Culturalmente as Unidades de Terapia Intensiva (UTI) remetem aos pacientes e familiares uma ideia de gravidade associada com a perda. Consequentemente, a internação nestes ambientes muitas vezes ocasiona momentos de estresse, sentimentos de incerteza, negação, desespero, culpa, ansiedade e até mesmo medo da morte do familiar interna$\mathrm{do}^{1-2}$. Neste sentido é necessário compreender a família como parte importante desse processo, no qual o paciente e a família devem ser vistos como uma unidade de cuidado, tendo em vista que a assistência prestada a um deles reflete no outro³.

No entanto, ressalta-se que nos ambientes de assistência ao paciente crítico, predominantemente a atenção dos familiares fica centrada na condição de ameaça à vida de seu parente e na evolução do seu estado de saúde. O que implica ações que produzem desconforto para com elas mesmas, como não se alimentar, não conseguir descansar, menosprezar os próprios problemas de saúde e verbalizar pouco suas angústias e medos ${ }^{4}$.

O conforto pode ser entendido de maneira geral como uma experiência subjetiva de natureza multidimensional que se modifica no tempo e espaço, dependendo dos objetos com os quais o individuo interage. É um fenômeno complexo, que depende do contexto social, econômico e cultural das pessoas, podendo ser caracterizado como um estado e/ou um sentimento, pois aborda questões biopsicossociais e ambientais ${ }^{5}$.

Destaca-se que, no ambiente hospitalar, a promoção do conforto vai além da esfera ambiental, abrange interações dos familiares com a competência técnico-científica, ética e humanística da equipe de saúde. E também é influenciada pela capacidade e estratégias de enfrentamento utilizadas por eles, sustentadas pelas suas experiências anteriores ${ }^{6}$.

Considerando-se a escassez de trabalhos que abordem essa temática na perspectiva da família e, entendendo o conforto deste grupo como uma das metas do cuidado de enfermagem torna-se indispensável aproximar-se da compreensão desse fenômeno, tendo como base o conforto e o desconforto gerado a eles durante o período vivenciado dentro do ambiente hospitalar. Destarte, no ano de 2014, Freitas validou a Escala de Conforto para Familiares de Pessoas em Estado Crítico de Saúde (ECONF). Uma escala nacional, desenvolvida para mensurar o nível de conforto desta população em especial?.

Acredita-se que, conhecer as causas de desconforto, pode fomentar ações que venham potencializar ações de conforto a esta clientela, dando oportunidades à equipe de criar espaços de discussões e compartilhamentos das dificuldades encontradas no ambiente de trabalho, para assim con- tribuir na humanização da assistência prestada aos familiares.

Diante do exposto, o presente estudo tem como objetivo verificar o nível de conforto na dimensão segurança de familiares de pacientes internados em leitos de Unidade de Terapia Intensiva

\section{MÉTODO}

\section{TIPO DE ESTUDO}

Trata-se de um estudo quantitativo, descritivo e transversal.

\section{LOCAL DO ESTUDO}

Realizado no período de junho a setembro de 2019 em dois serviços de terapia intensiva de uma Unidade de Cuidado Intensivo de um hospital universitário de grande porte da região sul do Brasil.

\section{PARTICIPANTES DO ESTUDO}

Os participantes foram 40 familiares de pessoas internadas, que atenderam aos seguintes critérios de inclusão: ter um familiar adulto na UTI com mais de 24 horas de internação; ter realizado pelo menos uma visita ao seu familiar; ter idade $\geq$ a 18 anos; e, estar em condições emocionais para responder aos questionamentos da pesquisa.

\section{COLETA DE DADOS}

A coleta de dados foi realizada por meio da aplicação de dois instrumentos, o primeiro composto por perguntas fechadas sobre dados referentes ao membro da família internado e dados sociodemográficos dos familiares entrevistados e, o segundo composto por 20 itens que fazem parte da dimensão segurança da ECONF

Para mensurar o grau de conforto em relação a cada item, foi utilizado uma régua-escala graduada do tipo Likert, com cinco intervalos de respostas, que variam entre, 1 - nada confortável, 2 - pouco confortável, 3 - mais ou menos confortável, 4 - muito confortável e 5 - totalmente confortável. Importante ressaltar que a escala de medida é crescente, deste modo, quanto maior o valor atribuido aos itens, maior é o grau de conforto do familiar de pessoas internadas em serviços críticos de saúde.

A partir do valor das respostas da ECONF considerou-se para a interpretação dos resultados: Pouco conforto até 4,19; Médio conforto de 4,20 a 4,59; Elevado conforto acima de $4,60^{7}$

\section{PROCEDIMENTOS DE ANÁLISE}

Os dados foram tabulados e armazenados em banco de dados no programa $E x c e l \Theta$, posteriormente foram transportados para o programa estatístico Statistical Package for the Social Sciences (SPSS) versão 19 e analisados por meio de estatística descritiva simples. 


\section{ASPECTOS ÉTICOS}

O projeto foi aprovado pelo Comitê de Ética em Pesquisa em Seres Humanos, sob o ParecerN N 3.320.581. Foram respeitadostodosospreceitos éticos de pesquisa em seres humanos ${ }^{8}$, de modo que todos os participantes foram esclarecidos sobre o objetivo do estudo, o sigilo ea possibilidade de declinar a participaçãona pesquisa a qualquertempo.

\section{RESULTADOS}

Dos 40 familiares entrevistados, $70 \%$ eram do sexo feminino, $45 \%$ encontravam-se na faixa etária de 40 a 59 anos, 52,5\% eram casados ou viviam com o companheiro (a), 55\% eram praticantes da religião católica, 42,5\% tinham o ensino médio completo,
$55 \%$ estavam trabalhando, 57,5\% eram parentes de primeiro grau, $55 \%$ não residiam com o paciente, $67,5 \%$ já tiveram outro familiar internado em UTI e 10\% apenas já tinha vivido a experiência de internação em UTI, conforme tabela 1.

Em relação aos pacientes internados, 55\% eram do sexo masculino, 50\% encontravam-se na faixa etária de 60 anos ou mais, $77,5 \%$ em condição clínica grave estável, 47,5\% em ventilação mecânica, 30\% com tempo de permanência acima de 15 dias na UTI e em relação ao sistema afetado que levou a internação $30 \%$ era devido a complicações neurológicas e 30\% respiratório, conforme apresentado na tabela 2 .

Tabela 1 - Caracterização sociodemográfica dos familiares das pessoas internadas. Curitiba, Paraná, Brasil, 2019

\begin{tabular}{|c|c|c|c|c|c|}
\hline \multirow{2}{*}{\multicolumn{2}{|c|}{ Caracterização sociodemográfica dos familiares }} & \multicolumn{2}{|c|}{ UTI I } & \multicolumn{2}{|c|}{ UTI II } \\
\hline & & $\mathbf{N}^{\circ}$ & $\%$ & $\mathbf{N}^{\circ}$ & $\%$ \\
\hline & & 22 & $55 \%$ & 18 & $45 \%$ \\
\hline \multirow{2}{*}{ Sexo } & Feminino & 15 & $68,2 \%$ & 13 & $72,2 \%$ \\
\hline & Masculino & 7 & $31,8 \%$ & 5 & $27,8 \%$ \\
\hline \multirow{4}{*}{ Idade $^{*}$} & 18 a 24 anos & 1 & $4,5 \%$ & 3 & $16,7 \%$ \\
\hline & 25 a 39 anos & 6 & $27,3 \%$ & 4 & $22,2 \%$ \\
\hline & 40 a 59 anos & 10 & $45,5 \%$ & 8 & $44,4 \%$ \\
\hline & $\begin{array}{l}60 \text { anos ou mais } \\
\text { Solteiro (a) }\end{array}$ & 5 & $\frac{22,7 \%}{36,4 \%}$ & $\frac{3}{6}$ & $\frac{16,7 \%}{33,3 \%}$ \\
\hline \multirow{3}{*}{ Estado Civil } & Casado (a) ou vive com o companheiro & 10 & $155 \%$ & 11 & $611 \%$ \\
\hline & & 10 & $45,5 \%$ & 11 & $01,1 \%$ \\
\hline & Separado (a)/divorciado(a) & $\frac{1}{7}$ & $4,5 \%$ & 0 & $0,0 \%$ \\
\hline \multirow{5}{*}{ Religião** } & $\begin{array}{l}\text { VIuvo (a) } \\
\text { Adventista }\end{array}$ & $\frac{5}{1}$ & $\frac{13,6 \%}{4,5 \%}$ & $\frac{1}{0}$ & $0,0 \%$ \\
\hline & Católica & 10 & $45,5 \%$ & 12 & $66,6 \%$ \\
\hline & Espírita & 0 & $0,0 \%$ & 2 & $11,1 \%$ \\
\hline & Evangélica & 9 & $40,9 \%$ & 3 & $16,7 \%$ \\
\hline & Sem religião & 2 & $9,1 \%$ & $\frac{1}{0}$ & $5,6 \%$ \\
\hline \multirow{6}{*}{ Escolaridade } & $\begin{array}{l}\text { Sem escolaridade } \\
\text { Ensino fundamental completo }\end{array}$ & 0 & $0,0 \%$ & 4 & $22,2 \%$ \\
\hline & Ensino fundamental incompleto & 3 & $13,6 \%$ & 0 & $0,0 \%$ \\
\hline & Ensino médio completo & 8 & $36,3 \%$ & 9 & $50 \%$ \\
\hline & Ensino médio incompleto & 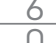 & $27,2 \%$ & $\frac{1}{7}$ & $\frac{5,6 \%}{167 \%}$ \\
\hline & $\begin{array}{l}\text { Ensino superior completo } \\
\text { Ensino superior incompleto }\end{array}$ & 1 & $4.5 \%$ & $\frac{3}{0}$ & $\frac{10,1 \%}{0,0 \%}$ \\
\hline & Pós-graduacão completa & 3 & $13.6 \%$ & 1 & $5.6 \%$ \\
\hline \multirow{5}{*}{ Trabalho } & Trabalhando & 13 & $59,1 \%$ & 9 & $50,0 \%$ \\
\hline & Afastado (a) do trabalho & 3 & $13,6 \%$ & 3 & $16,7 \%$ \\
\hline & $\begin{array}{l}\text { Aposentado (a) por tempo de trabalho/ } \\
\text { idade }\end{array}$ & 3 & $13,6 \%$ & 3 & $16,7 \%$ \\
\hline & Aposentado (a) por doença & 2 & $9,1 \%$ & 0 & $0,0 \%$ \\
\hline & Desempregado (a) & & $4,5 \%$ & 3 & $16,7 \%$ \\
\hline \multirow{3}{*}{ Grau de parentesco ${ }^{* \star *}$} & lo Grau & 14 & $63,6 \%$ & 9 & $50,0 \%$ \\
\hline & 2o Grau & 5 & $22,7 \%$ & 4 & $22,2 \%$ \\
\hline & $\begin{array}{l}\text { 30 Grau } \\
\text { Outros }\end{array}$ & $\frac{1}{2}$ & $9,1 \%$ & $\frac{4}{1}$ & $5,6 \%$ \\
\hline \multirow{2}{*}{ Reside com o paciente internado } & Não & 11 & $50,0 \%$ & 11 & $61,1 \%$ \\
\hline & Sim & 11 & $50,0 \%$ & 7 & $38,9 \%$ \\
\hline \multirow{2}{*}{$\begin{array}{l}\text { Experiência de estar internado em } \\
\text { uma UTI }\end{array}$} & Não & 20 & $90,9 \%$ & 16 & $88,9 \%$ \\
\hline & Sim & 2 & $9,1 \%$ & 2 & $11,1 \%$ \\
\hline \multirow{2}{*}{$\begin{array}{l}\text { Experiência de algum outro familiar } \\
\text { próximo internado em UTI }\end{array}$} & Não & 7 & $31,8 \%$ & 6 & $33,3 \%$ \\
\hline & Sim & 15 & $68,2 \%$ & 12 & $66,7 \%$ \\
\hline
\end{tabular}

*Definição adotada do PNAD - População por grupos de idade?

${ }^{* *}$ Religiões que foram citadas pelos entrevistados.

${ }^{* * *}$ Relação de parentesco compreendida no art. 1591 do Código Civil de $2002^{10}$.

**** Referem-se à nora/genro e cunhado (a). 
Tabela 2 - Caracterização dos pacientes internados. Curitiba, Paraná, Brasil, 2019.

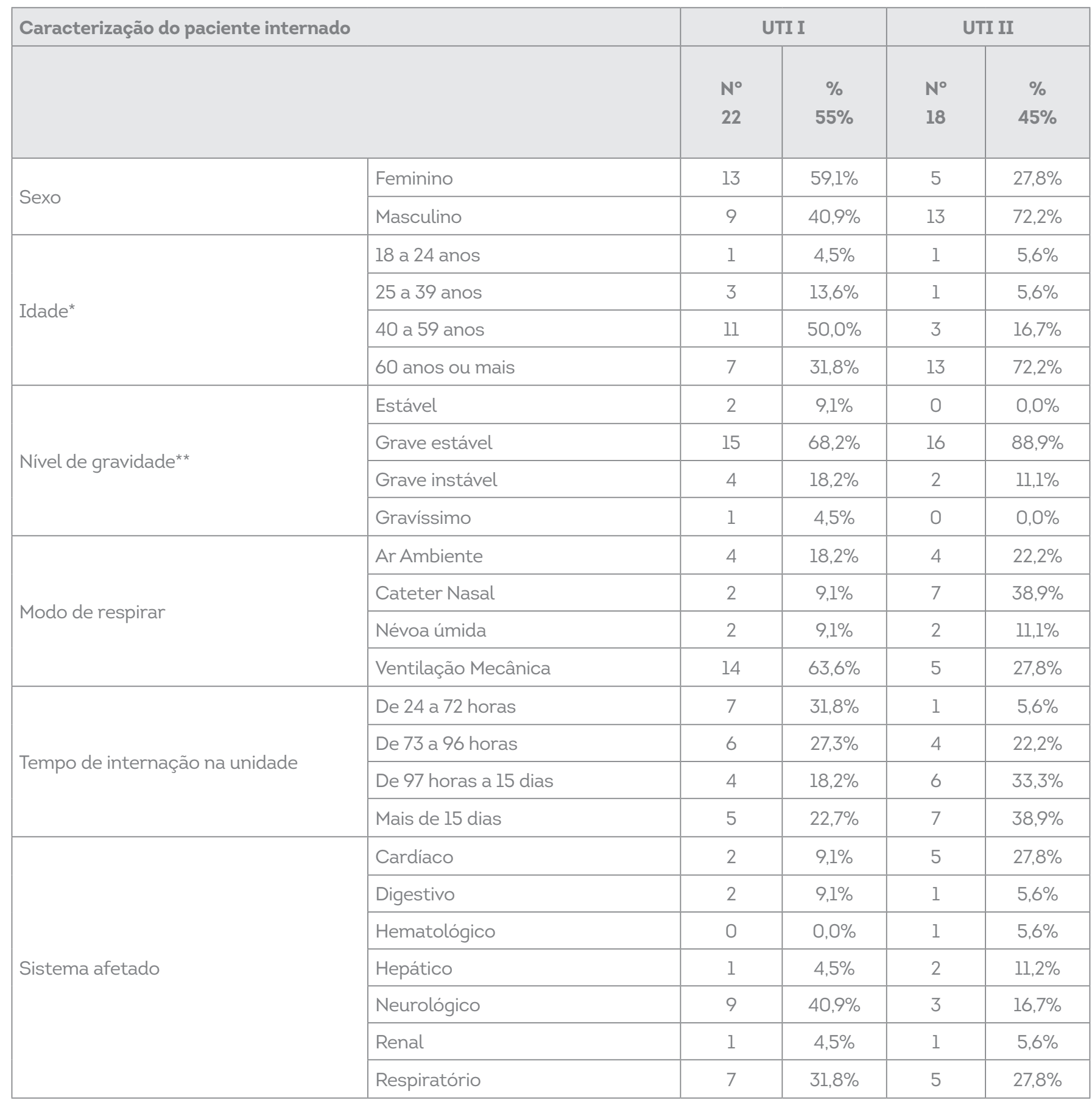

*Definição adotada do PNAD - População por grupos de idade?.

**Definição adotada para a variável nível de gravidade: 1. Estável - Cliente encontra-se na UTI, requerendo observação profilática do estado ventilatório e hemodinâmico; 2. Grave estável - Apresenta alteração em dados vitais, requer o uso de baixo nível de suporte para a manutenção de estado ventilatório, e/ou hemodinâmico, apresenta boa resposta a terapêutica instalada; 3. Grave instável - Apresenta alteração em dados vitais, requer o uso de alto nível de suporte para a manutenção de estado ventilatório, hemodinâmico, e/ou metabólico; 4. Gravíssimo - Apresenta alteração em dados vitais, requer o uso de alto nível de suporte para a manutenção de estado ventilatório e/ou hemodinâmico, entretanto não apresenta resposta satisfatória a terapêutica instaladall. 
Na tabela 3, está apresentada a média geral de conforto da dimensão segurança sendo 4,36 para UTI I e 4,31 para a UTI II. O item que mostrou maior nível de conforto está relacionado a percepção dos familiares de que a equipe se interessava pela recuperação do seu parente - 4,61, e o de menor nível de conforto ao item que se refere à receber uma palavra de apoio da equipe durante a internação na UTI - 4,11

Tabela 3 - Nivel de conforto dos familiares por itens da ECONF segundo a dimensão Segurança. Curitiba, Paraná, Brasil, 2019.

\begin{tabular}{|c|c|c|c|c|}
\hline PERGUNTA & $\begin{array}{l}\text { UTI I } \\
\text { Média }\end{array}$ & DP & $\begin{array}{l}\text { UTI II } \\
\text { Média }\end{array}$ & DP \\
\hline Sentir que a equipe se interessa pela recuperação do seu parente. & 4,32 & 0,48 & 4,61 & 0,50 \\
\hline Perceber que a equipe presta atenção às condições do seu parente. & 4,32 & 0,72 & 4,44 & 0,78 \\
\hline Receber uma palavra de apoio da equipe durante a internação na UTI. & 4,35 & 0,59 & 4,11 & 0,83 \\
\hline Saber qual tratamento está sendo dado ao seu parente. & 4,41 & 0,59 & 4,22 & 0,65 \\
\hline Ser atendido (a) com gentileza na recepção da UTI. & 4,32 & 0,65 & 4,33 & 0,59 \\
\hline Perceber tranquilidade no atendimento ao seu parente. & 4,18 & 0,59 & 4,28 & 0,67 \\
\hline Perceber que a equipe tem paciência para ouvir os familiares. & 4,27 & 0,55 & 4,28 & 0,67 \\
\hline Perceber que a equipe da UTI oferece informações com boa vontade. & 4,36 & 0,66 & 4,39 & 0,70 \\
\hline Perceber competência profissional naqueles que trabalham na UTI. & 4,41 & 0,59 & 4,44 & 0,62 \\
\hline $\begin{array}{l}\text { Perceber que os profissionais não insistem para que você saia logo ao término da } \\
\text { visita. }\end{array}$ & 4,36 & 0,58 & 4,33 & 1,03 \\
\hline Perceber que o seu parente recebe atendimento rápido quando necessário. & 4,32 & 0,78 & 4,22 & 0,94 \\
\hline Perceber que você é atendido (a) com tranquilidade pela equipe. & 4,32 & 0,48 & 4,33 & 0,69 \\
\hline Perceber que o seu parente tem recebido os cuidados de higiene. & 4,36 & 0,58 & 4,17 & 0,92 \\
\hline Receber informações dos profissionais de forma que você possa entender. & 4,48 & 0,60 & 4,17 & 0,86 \\
\hline Receber informação detalhada sobre a situação de seu parente. & 4,55 & 0,51 & 4,28 & 0,83 \\
\hline Saber que a melhor assistência possível está sendo dada ao seu parente. & 4,36 & 0,66 & 4,44 & 0,62 \\
\hline Ser tratado (a) gentilmente pelos profissionais da UTI. & 4,45 & 0,51 & 4,22 & 1,11 \\
\hline Saber que a UTI oferece segurança à recuperação do seu parente. & 4,45 & 0,51 & 4,39 & 0,61 \\
\hline $\begin{array}{l}\text { Perceber que os profissionais da UTI compreendem a situação que você está pas- } \\
\text { sando. }\end{array}$ & 4,27 & 0,77 & 4,28 & 0,83 \\
\hline Ter profissionais disponiveis para ajudar o seu parente. & 4,36 & 0,49 & 4,33 & 0,84 \\
\hline DOMÍNIO SEGURANÇA & 4,36 & 0,46 & 4,31 & 0,59 \\
\hline
\end{tabular}




\section{DISCUSSÃO}

Houve predomínio do sexo feminino na amostra dos participantes como familiares dos pacientes internados, isso pode ser devido ao aspecto cultural que conferiu a mulher o papel de cuidadora universal. Resultado semelhante ocorreu no estudo realizado em um hospital universitário de Belém-Pará que também constatou mais mulheres como acompanhantes no processo de hospitalização de pacientes internados em ambiente intensivo ${ }^{12-13}$.

Os parentes de primeiro grau foram os mais presentes, situação que pode ser motivada pela existência de vínculo afetivo, sentimento de compromisso e responsabilidade com o familiar internado ${ }^{14}$. Em relação ao estado civil dos familiares, constatou-se que a maioria eram casados ou viviam com o companheiro (a) e que durante aquele período estavam trabalhando, indicando que foi necessária adaptação à situação da hospitalização de seu ente. Isso corrobora o estresse familiar, uma vez que o acompanhante precisa dividir o seu tempo e se organizar para dar conta da nova rotina que envolve cuidados pessoais, atividades com a familia nuclear, o trabalho e os cuidados com o familiar hospitalizado. Estudo realizado em um hospital público no interior da Bahia sobre o cotidiano do familiar acompanhante durante a hospitalização de um membro da família, relata o desgaste físico e emocional a que esses familiares ficam expostos quando são responsáveis por tantas atividades ${ }^{15}$.

Sobre o familiar internado, o presente estudo demonstrou a prevalência do sexo masculino na faixa etária de 60 anos ou mais. Este dado pode ser resultado pela baixa procura dos homens aos serviços de saúde. Juntamente com o envelhecimento populacional, existe aumento da frequência de pacientes idosos nos serviços hospitalares. Os achados deste estudo, corroboram com pesquisa realizada com 975 prontuários, a qual evidenciou que $58,4 \%$ dos pacientes internados em uma UTI do estado do Rio Grande do Sul eram do sexo masculino, e o maior número de internações ocorreu com pessoas acima de 60 anos $^{16}$.

A dimensão segurança da ECONF, representa o conforto relacionado à confiança dos familiares na competência técnico-científica da equipe de saúde, bem como, a sua competência humanística que se relaciona à consideração da família pelos profissionais da instituição hospitalar e suas demonstrações afetivas $^{7}$

Os itens que menos pontuaram no nível de conforto, na tabela 3, considerados pouco confortáveis, estão relacionados a receber uma palavra de apoio da equipe durante a internação na UTI - 4,1l e receber informações dos profissionais de forma que você possa entender - 4,17.

A complexidade inerente a ambientes de cuidados intensivos, dificulta o desenvolvimento do cuidado humanizado, podendo distanciar os enfermeiros desses valores em seu cotidiano, isto pode estar relacionado ao fato de uma unidade de cuidados intensivos precisar de equipamentos de alta tecnologia para atender os pacientes em estado crítico de saúde, que necessitam ser monitorizados constantemente, e por vezes todo esse aparato tecnológico, vem de encontro com a humanização do cuidado que deve ser centralizada na assistência individualizada. Frequentemente, as rotinas diárias e todos esses equipamentos, acabam por mecanizar o cuidado e consequentemente a família acaba não sendo vista e nem incluída como sujeito dessas práticas de saúdel.

Estudo que teve como objetivo descrever como o enfermeiro se apropria do cuidado no acolhimento da família na UTI, evidenciou que apesar dos enfermeiros compreenderem a familia como uma unidade de cuidado, não se sentem preparados para esse atendimento. Esses profissionais relataram que sua atenção às famílias fica limitada a realização de rotinas burocráticas de coleta de dados na internação do paciente e, na atualização de informações sobre o estado clínico do paciente durante as visitas ${ }^{15}$

Os itens que mais pontuaram no nível de conforto, sendo considerados de médio a elevado conforto, estão relacionados a ser tratado gentilmente pelos profissionais - 4,45, sentir que a equipe se interessa pela recuperação do seu parente - 4,61 e saber que a UTI oferece segurança à recuperação do seu parente $-4,45$.

O sentimento de conforto ligado à segurança é garantido diante da ideia de que seu parente está recebendo um atendimento de qualidade, tanto no aspecto técnico e como humano. Quando se tem o entendimento de que a equipe de saúde atua em conjunto com aquele ambiente tecnológico e oferece a segurança de recuperação, esse estado de conforto é também difundido para as relações entre familiares e profissionais. Isso significa que o conforto decorre de práticas de cuidado que valorizem a humanidade associada às práticas cotidianas do trabalho ${ }^{4}$.

Neste sentido, a segurança é experienciada pela família quando estabelecida uma relação de confiança entre ela e os profissionais, quando os familiares percebem atitudes solidárias das pessoas que os atendem, quando expressam tranquilidade e compreensão pela situação enfrentada, e fornecem informações detalhadas e compreensiveis sobre as condições do parente internado 4 .

Os resultados positivos deste trabalho, com relação ao nível de conforto do familiar de pessoas internadas em ambientes críticos pode estar relacionado a satisfação dos usuários com os serviços prestados pela equipe de saúde de ambientes intensivos da instituição em que o estudo foi realizado. Destaca-se que, este hospital, faz parte de um projeto nacional do Ministério da Saúde que visa melhorar a segurança do paciente, denominado 
"Melhorando a Segurança do Paciente em Larga Escala no Brasil" onde uma das pautas é o acesso livre dos acompanhantes nos ambientes de terapia intensiva e a inclusão da família no cuidado ao paciente $\mathrm{e}^{17-18}$.

\section{Limitações do estudo}

Como limitação, neste estudo houve dificuldade durante a coleta de dados, pois os participantes tenderam a projetar suas respostas em situações desejadas e não com a situação vivenciada, apesar de ser explicado que as respostas deveriam ser dadas com relação a vivência deles durante aquele momento.

\section{Contribuições para a prática}

Entende-se que a família exerce papel de colaboradora no processo de tratamento e recuperação da saúde de seus entes. Desta forma, precisa ser considerada como uma parte integrante do cuidado da equipe de saúde, uma vez que a família é um sistema do qual o parente hospitalizado faz parte. Nesse sentido, constitui-se em objeto de cuidado da enfermagem, com uma série de demandas que precisam ser atendidas, como o acesso à informação sobre o estado de saúde do seu ente, e necessidades de cuidados consigo mesmo que contemplem seus aspectos físicos, psicológicos, espirituais, com acomodações adequadas que presem o seu conforto de maneira geral ${ }^{3-1}$.

\section{CONCLUSÃO}

O nivel de conforto na dimensão segurança da ECONF mostrou-se médio, evidenciando reflexos positivos da inclusão da família no contexto hospitalar relacionado ao acolhimento, interação com a equipe de saúde e confiabilidade no cuidado prestado ao seu familiar. Acredita-se assim, que a qualificação profissional e humanização no cuidado precisam ser cada vez mais fomentadas nas instituições hospitalares a fim de promover inserção da familia no plano terapêutico do paciente.

Contribuições dos Autores: Edineia Miranda Machado e Tatiana Brusamarello contribuíram em todas as etapas do estudo.

\section{REFERÊNCIAS}

1. Ariza LC, Hito PD. Estalella GM, Clavero GV, Canut TL, Garcia MR. Implementation of the evidence for the improvement of nursing care to the critical patient's family: a participatory action research. BMC Health services research. v. 18, n.357, 2018. Disponivel em: <https://www.ncbi.nlm.nih.gov/pubmed/29747635>. Acesso em: 25/02/2019.

2. Correio RAPPV, Vargas MAO, Carmagnani MIS, Ferreira ML, Luz KR. Desvelando Competências do Enfermeiro de Terapia Intensiva. Enferm Foco. V.6, n.1/4, p.46-50. 2016. Disponivel em: <http://revista.cofen.gov.br/index.php/enfermagem/article/view/576/258>. Acesso em: 18/01/2020.

3. Matos JC, Borges MS. A familia como integrante da assistência em cuidado paliativo. Revista de Enfermagem da UFPE on-line, [SI], v. 12, n. 9, p. 2399-2406, 2018. ISSN 1981-8963. Disponivel em: <https://periodicos.ufpe.br/revistas/revistaenfermagem/article/view/234575/29932 >. Acesso em: 03/12/2019.

4. Valente CO. Fonseca GM. Freitas KS, Mussi FC. Conforto familiar a um parente internado na unidade de terapia intensiva. Rev. baiana enferm. 31 (2): 17597. 2017. Disponivel em: <https://portalseer.ufba.br/index.php/enfermagem/article/view/17597>. Acesso em: 03/12/2019.

5. Coelho EOE. O conceito de conforto na estratégia saúde da familia. Rio de Janeiro, 2018. Disponivel em: <http://www.unirio.br/ppgenf/dissertacoes/ dissertacoes-ppgenf-unirio-ano-2018/dissertacao-elciana-de-oliveira>. Acesso em: 03/12/2019.

6. Ponte KMAP. Silva LF. Conforto como resultado do cuidado de enfermagem: revisão integrativa. Rev. pesqui. cuid. fundam (Online). V. 7. n. 2, p. 2603-26l4. 2015. Disponivel em: <http://bases.bireme.br/cgi-bin/wxislind.exe/iah/online/?IsisScript=iah/iah. xis\&src=google\&base=BDENF\&lang=p\&nextAction=InkEexprSearch=26918 indexSearch=ID> Acesso em: 03/12/2019.

7. Freitas KS. Construção e validação da escala de conforto para familiares de pessoas em estado crítico de saúde (ECONF). 2014. Tese (Programa de PósGraduação em Enfermagem) - Escola de Enfermagem da Universidade Federal da Bahia. 2014. Disponível em: < https://www.scielo.br/pdf/rlae/v23n4/ pt_0104-1169-rlae-23-04-00660.pdf>. Acesso em: 25/02/2019.

8. Brasil. Resolução no 466, de 12 de dezembro de 2012. Diretrizes e normas regulamentadoras de pesquisa envolvendo seres humanos. Diário Oficial da União, Brasilia, DF, 12 dez. 2012. Disponivel em: <http://www.conselho.saude.gov.br/resolucoes/2012/Reso466.pdf>. Acesso em: 12/03/2019.

9. Instituto Brasileiro de Geografia e Estatística. Pesquisa Nacional por Amostra de Domicilio Contínua Trimestral - População, por grupos de idade. Tabela 5918. 2019. Disponivel em: <https://sidra.ibge.gov.br/tabela/5918>. Acesso em: 20/03/2019.

10. Brasil. Código Civil - Lei n 10.406 de 10 de janeiro de 2002. Das relações de parentesco. Art. 1.591. Disponivel em: <https://www.soleis.adv.br/codigocivilnovo. htm>. Acesso em: 20/03/2019.

11. Consenso Brasileiro de Monitorização e Suporte Hemodinâmico. RBTI. v.17, n. 4, 2005. Disponivel em: <https://www.amib.org.br/fileadmin/user_upload/ amib/2018/junho/15/ConsensoMonitorizacaoSuporteHemodinamico.pdf>. Acesso em: 20/03/2019.

12. Meira EC, Reis LA, Gonçalves LHT, Rodrigues VP, Philipp RR. Vivências de mulheres cuidadoras de pessoas idosas dependentes: orientação de gênero para o cuidado. Esc. Anna Nery, Rio de Janeiro, v.21, n.2. 2017. Disponivel em: <http://www.scielo.br/scielo.php?script=sci_arttext\&pid=S1414-81452017000200217\&ln g=en\&nrm=iso>. Acesso em: 27/12/2019.

13. Reis EB, Branco JEB, Silva CSS, Torres RSC, Borges JOP, Moraes AD. "Se tem batimento, tem vida": vivências em torno da hospitalização na visão de familiares de pacientes internados no CTI. Revista Eletrônica Acervo Saúde, v. 11, n. 16, p. ell75. 2019. Disponivel em: <https://acervomais.com.br/index.php/saude/ article/view/1175/893>. Acesso em: 27/12/2019.

14. Beuter M, Brondani CM, Szareski C, Lana LD, Alvim NAT. Perfil de familiares acompanhantes: contribuições para a ação educativa da enfermagem. REME - Rev. Min. Enferm.; 13(1): 28-33. 2009. Disponivel em: <http://www.reme.org.br/artigo/detalhes/159>. Acesso em: 27/12/2019.

15. Passos SSS, Pereira A. Nitschke RG. Cotidiano do familiar acompanhante durante a hospitalização de um membro da familia. Acta Paul Enferm. 2015; 28(6): 539-45. Disponivel em: < http://www.scielo.br/pdf/ape/v28n6/1982-0194-ape-28-06-0539.pdf>. Acesso em: 27/12/2019.

16. Pauletti M, Otaviano MLPO, Moraes AST, Schneider DS. Perfil epidemiológico dos pacientes internados em um Centro de Terapia Intensiva. Aletheia v.50, n.1-2. p.38-46. 2017. Disponivel em: <http://www.periodicos.ulbra.br /index.php/aletheia/ article/ download>. Acesso em: 27/12/2019.

17. Silveira RS, Funck CR, Lunardi VL, Avila LI, Lunardi Filho WD, Vidal DAS. Percepção dos trabalhadores de enfermagem acerca da satisfação no contexto do trabalho na UTI. Enferm Foco. V.3, n.2, p. 93-96. 2012. Disponivel em: <http://revista.cofen.gov.br/index.php/enfermagem/article/view/262/150>. Acesso em: $18 / 01 / 2020$

18. Penido A. Ministério da Saúde lança projeto para qualificar segurança dos serviços prestados a pacientes em hospitais públicos. Brasilia: Ministério da Saúde, 2017. Disponivel em: <http://u.saude.gov.br/index.php/cidadao/principal/agencia-saude/29358-ministerio-da-saude-lanca-projeto-para-qualificarseguranca-dos-servicos-prestados-a-pacientes-em-hospitais-publicos>. Acesso em: 23/12/ 2019. 